\title{
Static Path Planning for Mobile Beacons to Localize Sensor Networks
}

\author{
Rui Huang and Gergely V. Záruba \\ Computer Science and Engineering Department \\ The University of Texas at Arlington \\ 416 Yates, 300NH, Arlington, TX 76019 \\ Email: rxh1725@omega.uta.edu, zaruba@uta.edu
}

\begin{abstract}
In this paper, we study the static path planning problem with wireless sensor network localization as the primary objective. We consider a model in which sensors are assumed to be uniformly deployed to a predefined deployment area. We then deploy a robot to serve as a mobile beacon to enable the localization of the sensor nodes. The robot follows a pre-determined static path while periodically broadcasting its current location coordinates to the nearby sensors. The static path planning problem looks for good paths that result in better localization accuracy and coverage of the sensor network while keeping the path length bounded. We propose two new path types, CIRCLES and S-CURVES, that are specifically designed to reduce the collinearity during localization. We compare our solution with existing ones using the Cramer Rao Bound (CRB) as the evaluation tool, which gives an unbiased evaluation regardless of localization algorithm used. The evaluation shows that our solutions cope with collinearity in a more effective manner than previous solutions. Our solutions provide significantly better localization accuracy and coverage in the cases where collinearity is the greatest problem.
\end{abstract}

\section{Introduction}

Wireless sensor networks are expected to be the basic building block of pervasive computing environments. To realize wireless sensor networks in large scale, location discovery is emerging as an important task as it has been observed and shown that (semi-) accurate location information can greatly improve the performance of other tasks such as routing, energy conservation, or maintaining network security. One method to complete the location discovery is to employ a GPS-equipped robot as a mobile beacon to the already deployed sensor network. In this method, the robot travels through the deployment area while broadcasting its location along the way. Sensors localize themselves by monitoring information coming from the beacon. Other than serving as the mobile beacon, the robot can perform other tasks necessary for the operation of sensor networks. For instance, the robot could be used to reconfigure or recalibrate sensors, synchronize the clocks, collect data from the sensors (in an efficient manner), deploy new sensors, and disable existing ones. Thus, in sensor networks that already incorporate mobile robots as part of the design, enabling localization through the mobile beacon can be a cost-effective way of achieving sensor network localization.

In this paper, we consider the path planning problem of the mobile beacon. We ask the question of what should be considered as a better path to be taken by the mobile beacon with sensor localization as our primary objective. We study the problem of static path planning for localization prior to the beacon deployment. For this problem, we assume that, although we do not know the exact sensor locations prior to the beacon deployment, the sensors are uniformly distributed over a predefined deployment area. The objective of our static path planning is to design a path to guide the mobile beacon such that i) a higher percentage of the sensors can be localized (i.e., better coverage), ii) the localization error is minimized (i.e., better accuracy), and iii) the path length the mobile beacon travels is shortened. We consider such path planning as static since it is done prior to the localization and cannot be modified during the localization. As such, the static path planning problem is invariant to the actual localization algorithm.

Our main contributions in this paper include two new static path types, CIRCLES and S-CURVES, that are designed with localization accuracy and coverage in mind. We also introduce the concept of using Cramer Rao Bound (CRB), an unbiased lower bound of localization accuracy, as the evaluator of path types. We then compare our path types to previously proposed ones using the CRB analysis. Our results shows a clear advantage of our path types over others in terms of coverage and accuracy without increasing the path length. 


\section{Related Works}

Note that the path planning problem we are considering here is related but ultimately different than the path planning and localization problem in robotics. The problem in the field of robotics deals with the issue of localizing and navigating robots in an unknown environment based on a certain type of signal behavior (from a pre-calibrated RSSI map or pre-localized sensors) [1-3]. The problem we are considering here deals with localizing the sensors using a GPS-equipped mobile robot.

There has been limited amount of previous work on the path planning problem for sensor network location. The problem was first brought up by [4] where the authors were primarily interested in the localization algorithm design of mobile beacons. As a side note, the author acknowledged the difficulty in selecting an optimal trajectory for the beacon in that the trajectory needs to provide at least three noncollinear beacon broadcast locations for each sensor. However, the authors did not provide any specific path planning method.

While the authors of [5] did not explicitly study the path planning problem, they implicitly considered this issue during their localization algorithm design. In particular, they provided an algorithm running on the sensor nodes that tracks the series of beacon broadcasts to only use the noncollinear ones to localize. They do not introduce a path planning algorithm, i.e., the mobile beacon is assumed to move randomly, thus their method provides a passive way of extracting usable coordinates from the random path.

In [6], the authors studied three different types of static paths: SCAN, DOUBLE SCAN, and HILBERT in relationship to localization. The result shows that HILBERT has the best localization performance due to as its larger number of direction changes effectively reduces the collinearity during localization. Their results are obtained using simulations of randomly deployed sensor networks using a Monte Carlo localization (MCL) method [7] as the localization algorithm.

In this paper, we build on the work by [6] to design additional static paths that further reduce the collinearity. Instead of evaluating paths based on a particular localization algorithm, we use the more general Cramer Rao Bound (CRB) as the metric to compare different paths. The CRB gives a theoretic lower bound on the best localization error achievable by any localization algorithm. Thus, by using $\mathrm{CRB}$ as the metric, we are able to provide a fairer comparison by eliminating any bias introduced in favor of a particular localization algorithm. Under the same test case, a path type that achieves the better overall CRB should be considered to be better.

\section{Cramer-Rao Bound (CRB)}

In this paper, we use the Cramer-Rao Bound (CRB) as the evaluator of static path types. The CRB is an effective measure to qualify the localization inaccuracy attributed to the measurement types and noises [8]. The CRB is a lower bound on the covariance of any unbiased location estimator that uses noisy measurements such as RSSI, TOA, or AOA. Thus, the CRB provides a lower bound on the estimation accuracy of a given network scenario regardless of the localization algorithm. In other words, with CRB we have a way to tell how good the best any localization algorithm can do given a particular network, measurement type and measurement noise scenario. The CRBs of individual measurement types such as RSSI, TOA and AOA under most common noise models (mostly Gaussian) are discussed in more detail in [8].

Figure 1 shows the Cramer Rao Bound (CRB) of a single node to be localized based on an RSSI measurement model. Here, we use the signal propagation model and noise model given in [9]. In particular, we assume that the range measurement noise is Gaussian with a constant variance introduced by the shadowing. The received signal strength from a beacon location $v$ to a regular node $e$ that are $d_{v, e} \mathrm{~m}$ apart is therefore

$$
N\left(P_{0}-10 n_{p} \log _{10}\left(d_{v, e} / d_{0}\right), \sigma_{d B}^{2}\right)
$$

where $P_{0}$ is the received signal strength at a reference distance $d_{0}$. Here, we use $d_{0}=1 m . n_{p}$ is the path loss exponent that is environment-dependent, and $\sigma_{d B}^{2}$ is the constant variance introduced by the shadowing. As in [9], we choose $n_{p}=2$ and $\sigma_{d B}^{2}=1.7$.

To obtain data for Figure 1 we measured the CRB of any location within a $2 \mathrm{~m}$ by $2 \mathrm{~m}$ square, with three beacons placed at an inner circle of radius 0.5 centered at $(1,1)$. The three beacons are placed with equal angles at $(1+\cos (0) / 2,1+\sin (0) / 2),(1+\cos (2 / 3 \pi) / 2,1+$ $\sin (2 / 3 \pi) / 2)$, and $(1+\cos (4 / 3 \pi) / 2,1+\sin (4 / 3 \pi) / 2)$. It is clear from Figure 1 that the locations within the beacon deployment radius have lower CRBs. When the location of the node is outside the circle of beacons, the CRB progressively becomes greater; this is consistent with the intuition that it is more difficult to localize nodes outside the convex hull formed by the beacon locations.

\section{Static Paths}

In this section, we define four different static path types and compare the localization performance of them using the CRB analysis. As in [6], we adopt a deployment area consisting of a $480 \mathrm{~m}$ by $480 \mathrm{~m}$ square. To calculate the CRB, we use the values defined in the previous section. We test 


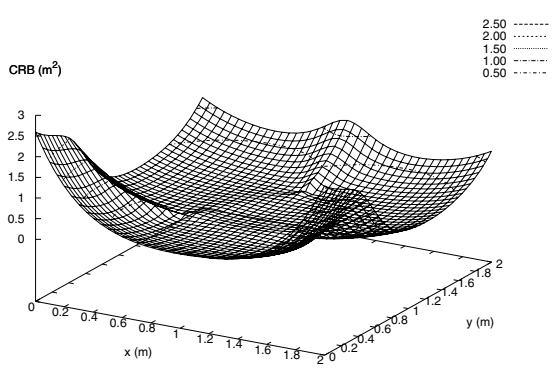
Figure 1. The Cramer Rao Bound (CRB) of
RSSI measurements.

four types of static paths: SCAN, HILBERT, CIRCLES and S-CURVES. Of the four types, SCAN and HILBERT were originally proposed in [6] (we include them for comparison purposes). We omit DOUBLE SCAN here as it has been shown in [6] that it does not provide a significant advantage over SCAN or HILBERT.

For sensor network localization using a mobile beacon, the CRB values of the sensors are influenced by the following factors:

1. Path Length. A longer path means that the mobile beacon would have more opportunity to broadcast its location. Thus, we expect a better overall localization (i.e., lower CRB) with a longer path.

2. Broadcast Interval. A shorter broadcast interval means that the mobile beacon would broadcast its location more frequently. Thus, we expect a better overall localization with a shorter broadcast interval.

3. Broadcast Range. A larger broadcast range (transmission radius) of the mobile beacon would allow each broadcast to cover more sensors. Thus, we expect a better overall localization with a larger broadcast range.

The four types of static paths considered here are shown in Figure 2. The solid lines show the path; the rectangles show the broadcast location; the small dots show the locations where localization can be performed. To provide a valid comparison among them, we fix the broadcast range $(45 \mathrm{~m})$. We set the broadcast interval to $10 \mathrm{~m}$ (i.e., there is one broadcast every 10 meters traveled), therefore the actual number of broadcast locations is a function of the path length. Each of the four different path types has a different path length. The solid line in Figure 2 shows the actual path, and the rectangles along the path show the broadcast locations (the distance between two rectangles is the broadcast interval). The small dots represent the locations from
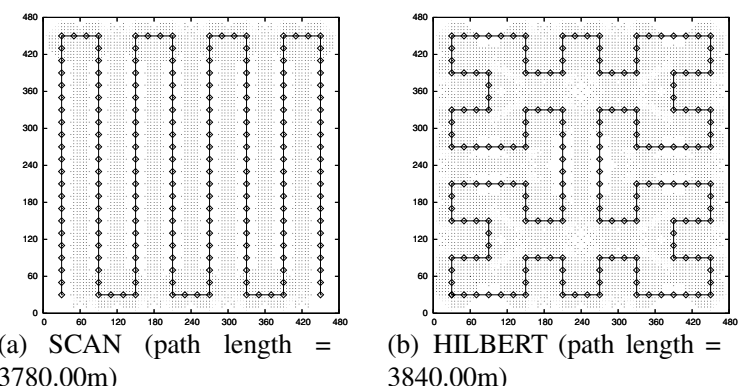

(b) HILBERT (path length $=$ $3840.00 \mathrm{~m})$

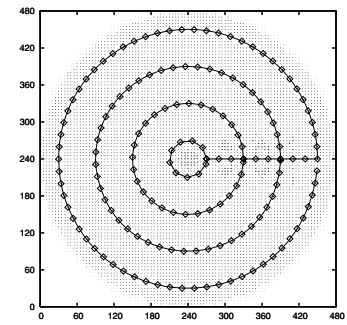

(c) CIRCLES (path length $=$ $3195.93 \mathrm{~m})$

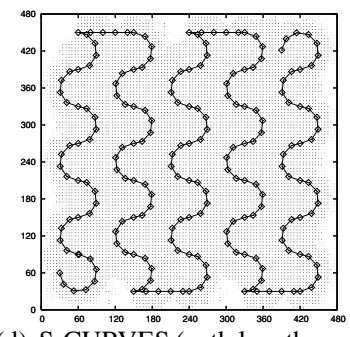

(d) S-CURVES (path length = 3752.92m)

Figure 2. Static Path Planning.

where a valid localization can be performed based on the RSSI range readings. Because of geometric constraints, a valid localization can be performed at a location only when at least three non-collinear broadcasts are heard.

\subsection{SCAN}

Of the four path types, SCAN, introduced in [6], is the most straight-forward in which the mobile beacon simply sweeps the deployment area in straight lines from left to right. More formally, SCAN divides the square deployment area into $n$ by $n$ sub-squares ( $n=8$ in our case) and connects their centers using straight lines as in Figure 2(a). The resolution, $R$, of SCAN is defined as the side length of each sub-square ( $R=60 \mathrm{~m}$ in our case). The drawback of SCAN is that straight lines introduce collinearity, and there are many locations where the beacon broadcasts heard are collinear. Since the broadcast range is set to $45 \mathrm{~m}$ and two nearby vertical lines in Figure 2(a) are two resolutions apart $(120 \mathrm{~m})$, the area near the vertical lines cannot be localized because all beacon broadcasts come from the same vertical line and thus are collinear. To reduce collinearity, we would have to reduce the resolution to match the broadcast range, which would substantially increase the path length.

\subsection{HILBERT}

To reduce the collinearity without significantly increasing the path length, HILBERT is proposed in [6], which 
makes the mobile beacon to take more turns. Same as SCAN, HILBERT divides the 2-dimensional space into $n$ by $n$ sub-squares ( $n=8$ in our case), but connects the centers of the sub-squares using $n$ line segments as shown in Figure 2(b). The resolution of HILBERT is defined as the side length of each sub-square ( $R=60 \mathrm{~m}$ in our case). While the path length of HILBERT is $n R$ longer than that of SCAN at the same resolution, it contains shorter line segments, which reduces collinearity. We can see in Figure 2(b) that a significantly greater area can be localized with HILBERT compared to SCAN.

\subsection{CIRCLES}

Since straight lines invariably introduce collinearity, we would like to reduce the amount of straight lines on the beacon path. Thus, we design CIRCLES which consists of a sequence of concentric circles centered within the deployment area. We define the resolution, $R$, of CIRCLES as half of the radius of the innermost circle, and we sequentially increase the radius by $R$ at each outer circle ( $R=60 \mathrm{~m}$ in our case). Note that we could define a spiral like path that looks like CIRCLES, but we decided to study CIRCLES because its resolution parameter is comparable to other path types.

As shown in Figure 2(c), since CIRCLES does not introduce collinearity, all areas within the circles can be localized. However, since the deployment area is a square, CIRCLES would not cover the four corners effectively without adding larger circles, which would increase the path length. Furthermore, CIRCLES has an inherent scalability issue. When the deployment area increases, CIRCLES would require the beacon path to contain larger circles. As the circles become larger, the amount of non-collinearity reduces, which in turn reduces the localization accuracy. Figure 3 illustrates such scalability issue in terms of CRB. Here, we measure the CRB of CIRCLES at various $y$ locations when fixing $x=240 m$ (i.e., splitting the deployment area in the middle). We observe that the CRB is at the minimum at the inner circle (around $240 \mathrm{~m}$ ), but it increases gradually to the outer circles (approaching $0 \mathrm{~m}$ to the left and 480m to the right.

\subsection{S-CURVES}

S-CURVES is based on SCAN, which progressively scans the deployment area from left to right. However, at each scan, S-CURVES takes an ' $S$ ' curve instead of going in a straight line. More formally, dividing the deployment square into $n$ by $n$ sub-squares $(n=8)$, and let the resolution of S-CURVES be $R(R=60 \mathrm{~m})$. Then, each vertical S curve consists of $n-1$ half squares of radius $\frac{R}{2}$, and there are a total of $\lfloor 2(n-1) / 3\rfloor+1 \mathrm{~S}$ curves from left to right. The $\mathrm{S}$ curves are connected with short straight lines like in

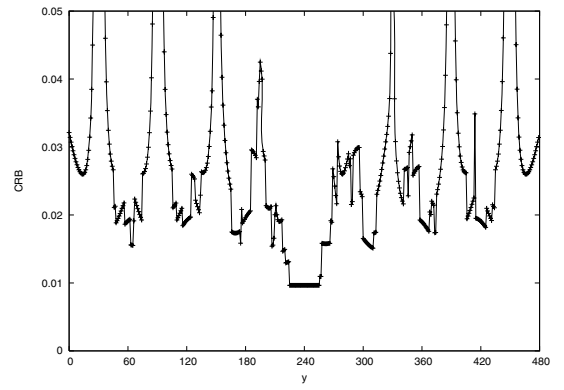

Figure 3. The CRB of CIRCLES at $x=240$.

SCAN.

\section{Evaluation}

We compare the path types based on three factors: i) total path length, ii) the localization coverage, and iii) the localization accuracy. Consider the path length first. For each of the four path types, the total path length, $L$, is a function of $R$ and $n$ :

$$
\begin{aligned}
L_{S C A N}= & \left(n^{2}-1\right) R \\
L_{H I L B E R T}= & n^{2} R \\
L_{C I R C L E S}= & \frac{n^{2} \pi R}{4}+\left(\frac{n}{2}-1\right) R \\
L_{S-C U R V E S}= & \frac{(n-1) \pi R}{2} \cdot(\lfloor 2(n-1) / 3\rfloor+1) \\
& +(n-2) R+\frac{R \pi}{2}
\end{aligned}
$$

As seen in our test scenario, CIRCLES has the shortest path length. The other three path types have similar path length, with S-CURVES being slightly shorter than the other two.

Now consider the localization coverage and accuracy. Here, we compare the four path types using the Cramer Rao Bounds (CRB). For each path type, we calculate the CRB at various locations of the entire deployment area. We assume a 1-hop propagation of RSSI readings, and thus the CRB at each location is a strict function of the RSSI readings from broadcast locations 1-hop away. For those locations that cannot be localized because of the unavailability of three non-collinear broadcast locations, the CRB will be infinity. For other locations that can be localized, the CRB gives a tight lower bound of the localization error that can be possibly achieved at the particular location. Thus, the CRB analysis gives an estimate of both localization coverage and localization accuracy.

To perform the CRB analysis, we divide the $480 \mathrm{~m}$ by $480 \mathrm{~m}$ deployment area into a system of $1 \mathrm{~m}$ by $1 \mathrm{~m}$ grids. We then calculate the CRB at every grid location and construct a histogram the CRB ranges produced by each of the 
four static path types. To study the relationship between the transmission range and the path resolution, we vary the beacon transmission range from $30 \mathrm{~m}$ to $75 \mathrm{~m}$, while fixing the path resolution at $60 \mathrm{~m}$. Notice that varying the transmission range while fixing the path resolution can be seen as equivalent to varying the path resolution while fixing the transmission range.

Figure 4 shows the results of our CRB analysis. For each of the transmission ranges, we construct a histogram consisting of seven categories based on the CRB ranges: $[0,0.01),[0.01,0.02),[0.02,0.03),[0.03,0.04)$, $[0.04,0.05),[0.05,0.5)$, and $[0.5$, inf $)$, represented as patterned boxes from bottom to top within the histogram bar. Each category contains the percentage of the grid locations whose CRB falls into its corresponding range. The percentage of a category is reflected by the size of the corresponding patterned box. Using the histogram, we are able to compare the static path types in terms of localization coverage as well as localization accuracy. A histogram with a large number of grid locations in the $[0.5$,inf) category (i.e., at the top of the histogram bar) indicates a poor coverage because the CRB values within this category are large indicating that those grid locations are difficult to localize. Conversely, a large number of grids in the first several categories (i.e., at the bottom of the histogram bar) indicates better localization accuracy.

Figure 4(a) clearly shows the superiority of CIRCLES and S-CURVES over the other two methods when the path resolution is higher than the transmission range. In such cases, many grid locations can only hear consecutive beacon broadcasts from the a single path segment. Since a majority of the path segments within SCAN and HILBERT are straight lines, collinearity becomes a major obstacle. On the other hand, CIRCLES and S-CURVES have a minimal number of straight line segments, and thus they cause much less amount of collinearity during localization. The result indicates the paths taken by CIRCLES and S-CURVES cover larger effective ground in terms of localization than SCAN and HILBERT. Another way to look at this is that it would take a longer path (for instance, using a smaller resolution) for SCAN and HILBERT to provide the equivalent coverage.

By increasing the transmission range (Figure 4(b) through 4(d)), the amount of collinearity reduces, and thus we observe performance improvement of SCAN and HILBERT. In those cases, S-CURVES still performs as well as SCAN and HILBERT. However, CIRCLES has the worst performance in Figure 4(d). This is primarily due to the fact that we use a square as our test deployment area, and thus leave the four corners uncovered by CIRCLES. As such, we also note that CIRCLES has significantly shorter path length than the other three path types. Thus, CIRCLES is not best suited for the square or rectangle deployment area.
But we can expect that it would work much better when the deployment area resembles a circle.

\section{Conclusion and Future Work}

In this paper, we studied the static path planning problem for wireless sensor network localization in pervasive computing. We presented two new static path types: CIRCLES and S-CURVES that are designed with better localization accuracy and coverage in mind. We have also proposed a new path evaluator using the unbiased Cramer Rao Bound (CRB). Using the CRB analysis, we showed that the path types proposed by us handle the collinearity problem much better. When the path resolution is much larger than the transmission range, the collinearity becomes more significant. In such cases, our solutions significantly outperform previously proposed path types.

The static paths work well when the sensors are assumed to be uniformly deployed. However, in the cases where such assumption is not valid, static path planning might not be the best solution, since the mobile beacon would attempt to cover the entire deployment area uniformly, including those parts where no sensor resides. Thus, there is a strong incentive to dynamically adjust the path during the localization procedure (dynamic path planning).

\section{References}

[1] G. V. Záruba, M. Huber, and F. A. Karmangar, "Monte Carlo Sampling Based In-Home Location Tracking with Minimal RF Infrastructure Requirements," in Proc. of IEEE GLOBECOM'04, vol. 6, pp. 3624-3629, December 2004.

[2] D. Kurth, "Range-Only Robot Localization and SLAM with Radio," Tech. Report CMU-RI-TR-04-29, Robotics Institute, Carnegie Mellon University, May, 2004.

[3] A. Georgiev and P. K. Allen, "Localization Methods for a Mobile Robot in Urban Environments," IEEE Transactions on Robotics, vol. 20, no. 5, pp.851-864, October 2004.

[4] M. L. Sichitiu and V. Ramadurai, "Localization of Wireless Sensor Networks with a Mobile Beacon," in Proc. of the First IEEE Conference on Mobile Ad-hoc and Sensor Systems (MASS 2004), pp. 174-183, Fort Lauderdale, FL, October 2004.

[5] K.-F. Ssu, C.-H. Ou, and H.C. Jiau, "Localization with Mobile Anchor Points in Wireless Sensor Networks," IEEE Transactions on Vehicular Technology, vol. 54, no. 3, pp. 1187-1197, May 2005. 


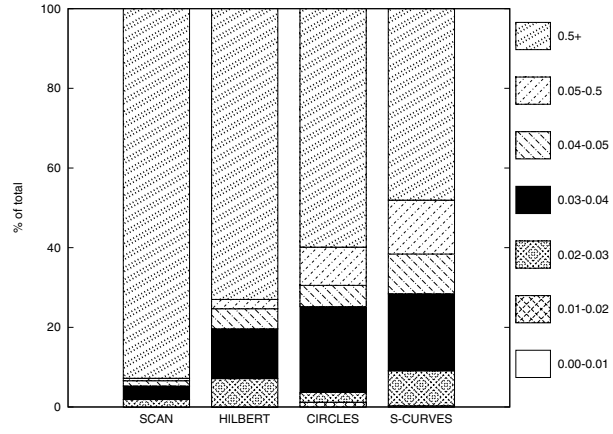

(a) Transmission Range $=30 \mathrm{~m}$

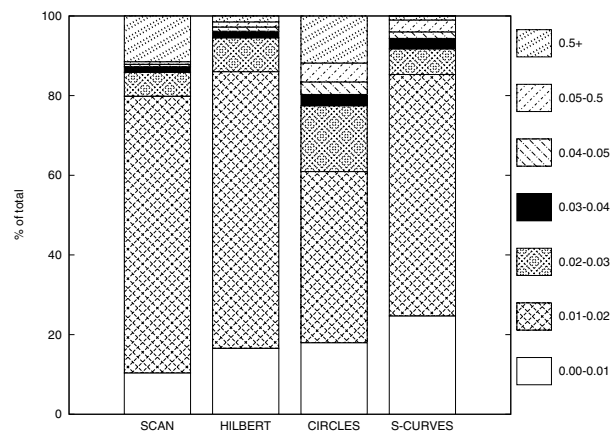

(c) Transmission Range $=60 \mathrm{~m}$

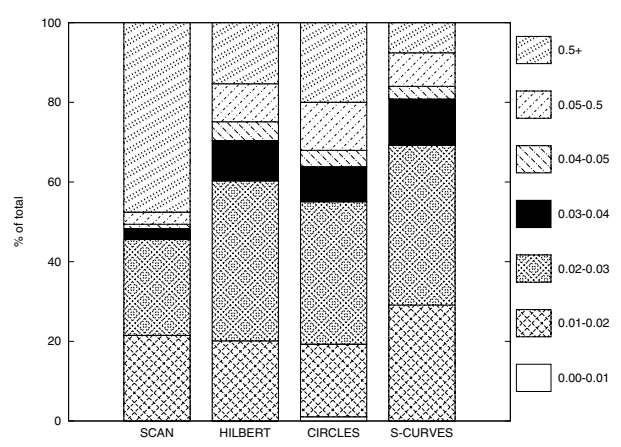

(b) Transmission Range $=45 \mathrm{~m}$

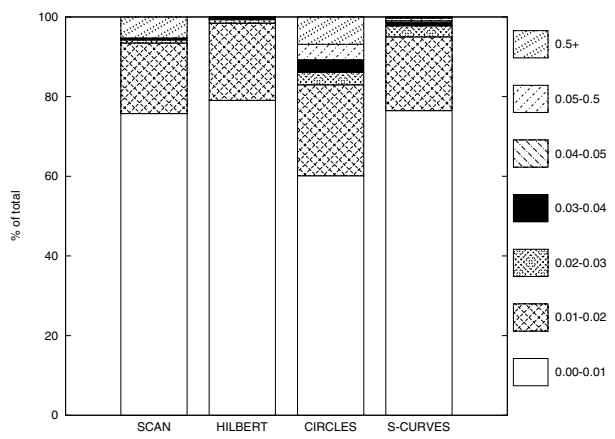

(d) Transmission Range $=75 \mathrm{~m}$

Figure 4. CRB ranges of the four static path types.

[6] D. Koutsonikolas, S. M. Das, and Y. C. Hu, "Path Planning of Mobile Landmarks for Localization in Wireless Sensor Networks," in Proc. of IEEE Distributed Computing Systems Workshops, pp. 86-86, 2006.

[7] L. Hu and D. Evans, "Localization for Mobile Sensor Networks," in Proc. of ACM MOBICOM'04, pp. 45-57, 2004.

[8] N. Patwari, A. Hero, J. Ash, R. Moses, S. Kyperountas, and N. Correal, "Locating the Nodes: Cooperative Geolocation of Wireless Sensors," IEEE Signal Processing Magazine, vol. 22, no. 4, pp. 54-69, July 2005.

[9] N. Patwari, A. O. Hero III, M. Perkins, N. S. Correal, and R. J. O'Dea, "Relative Location Estimation in Wireless Sensor Networks," IEEE Transactions on Signal Processing, vol. 51, no. 8, pp. 2137-2148, August 2003. 